\title{
Acute kidney injury in patients admitted with COVID-19 in a south Birmingham trust
}

\author{
Authors: Seema Jham, ${ }^{\mathrm{A}}$ Taimoor Shafiq, ${ }^{\mathrm{A}}$ Francesco Loverre, ${ }^{\mathrm{A}}$ Josh Edwards, ${ }^{\mathrm{A}}$ Megan Fahy, ${ }^{\mathrm{A}}$ Lorraine Harper, ${ }^{\mathrm{A}}$ \\ Jyoti Baharani ${ }^{\mathrm{A}}$ and Helen Eddington ${ }^{\mathrm{A}}$
}

\section{Introduction}

Several studies from China and the USA have shown high rates of acute kidney injury (AKI) in patients with COVID-19. A proportion of patients develop severe AKI and require renal replacement therapy (RRT).

The West Midlands saw a peak of COVID-19 around the months of March 2020 and April 2020. The University Hospitals Birmingham NHS Trust, which serves a population of around 2.2 million people, was one of the worst-hit trusts in the UK, with the one of the highest admission rates of COVID-19infected patients in those peak months.

The aim of our project was to determine the incidence of AKI in patients requiring admission for COVID and to compare outcomes between those patients with and without AKI.

\section{Methods}

We retrospectively analysed data from all patients that tested positive for COVID-19 from 1 March 2020 to 30 April 2020 and required admission into hospital.

Demographic data, laboratory data and other clinical information were extracted from the electronic medical record system. AKI was defined according to the KDIGO criteria and the stage of AKI was determined using the peak serum creatinine level after AKI detection. All data were analysed using SPSS Version 26.

\section{Results}

2,806 patients were identified with a positive PCR test from a sputum or nasopharyngeal swab at our trust in the above defined time periods. After exclusion of anyone under the age of 16 years, patients on replacement therapy or with a working kidney transplant and anyone not requiring admission, the details of 2,325 patients were analysed.

$55.2 \%$ of the patients were male, with $64.8 \%$ Caucasian, 14.7\% Asian/Asian British and the rest either of mixed race, Black, Chinese or unknown. Average age of patients admitted

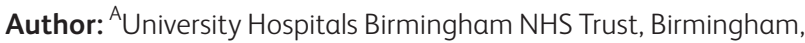
UK

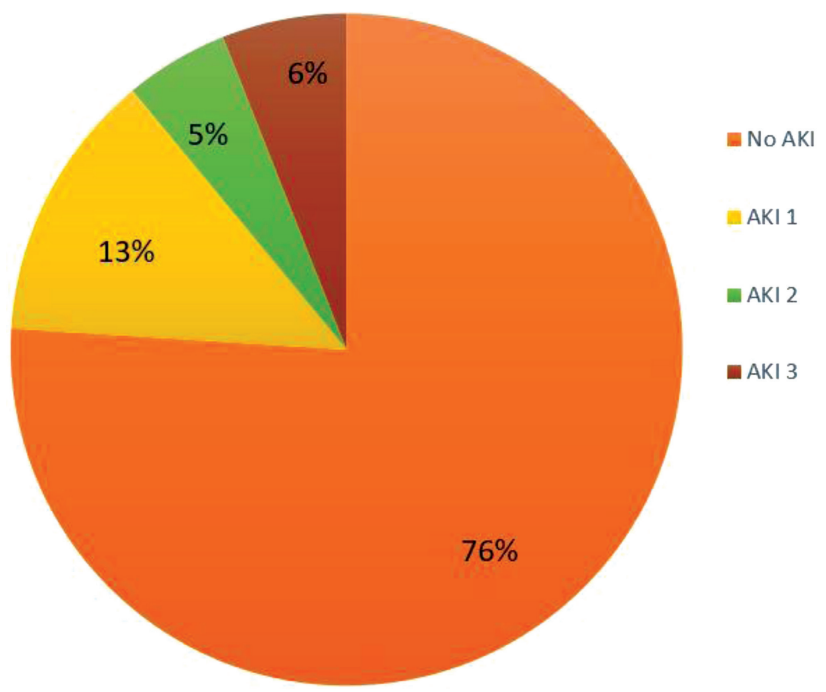

Fig 1. Proportion of admitted patients with acute kidney injury (AKI) from 7 days prior to the positive COVID-19 swab to any time before discharge.

was $69.48 \pm 18.28$ years and average length of stay was 9.95 \pm 11.26 days.

Of the 2,325 patients admitted, 553 patients (23.7\%) had an AKI of any stage from 7 days prior to the positive COVID-19 swab to any time before discharge (Fig 1).

Of the 553 patients with AKI, $11.75 \%$ (65 patients) required RRT in the form of continuous renal replacement therapy or intermittent haemodialysis. Only $38.3 \%$ of the patient with AKI 3 required RRT. Of the 65 patients who required RRT, only two are currently still on haemodialysis. Mortality was $41.5 \%$ in patients who required RRT.

A Kaplan-Meier survival analysis stratified by AKI status is shown in Fig 2. Patients with AKI 3 had a significantly lower survival than patients with AKI 2, with the trend continuing for patients with AKI 1 and patients with no AKI (log-rank p value $<0.01)$.

\section{Conclusion}

Although south Birmingham saw a large number of COVID cases requiring inpatient stay in the early pandemic of 2020, our 
Fig 2. Kaplan-Meier survival analysis stratified by acute kidney injury (AKI) status.

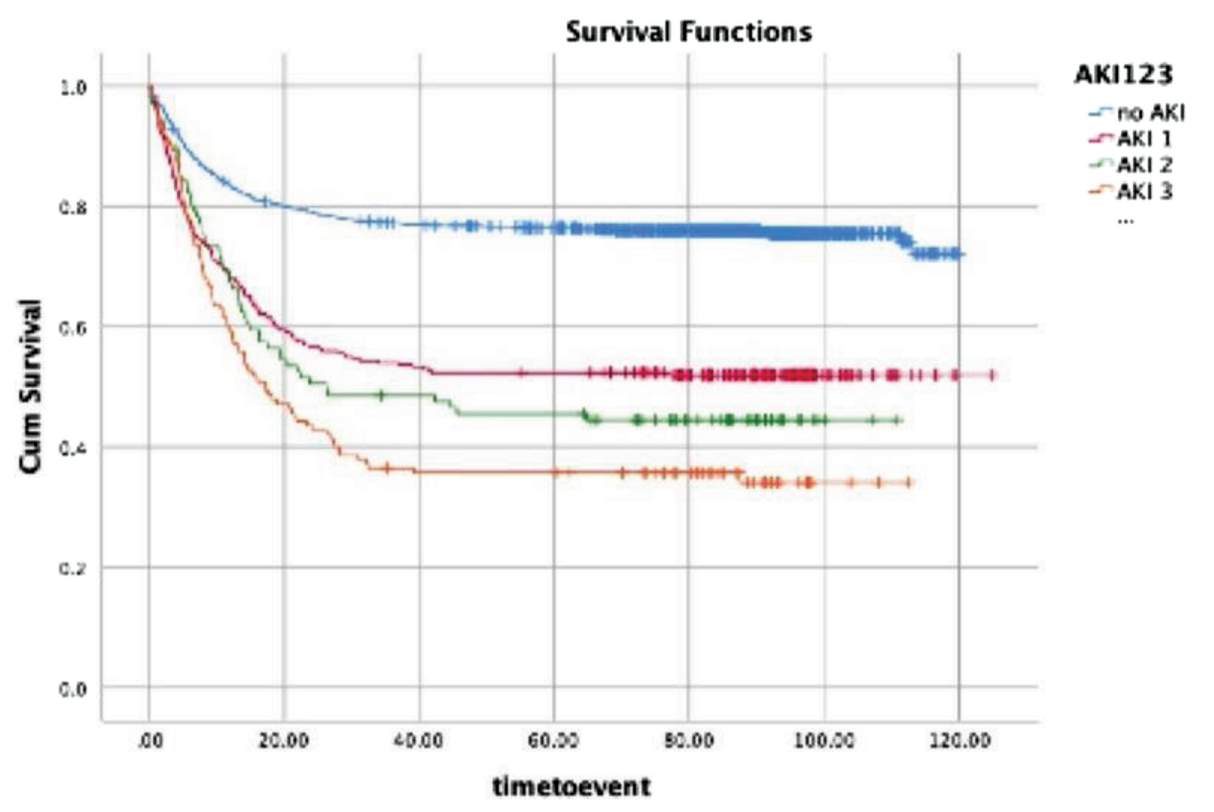

incidence of AKI has not been as high as that reported in the USA and China. As expected survival was worse in those with an AKI stage 3, but recovery from an AKI due to COVID 19 was also high, with only a minority of patients remaining on long term RRT (over 3 months after RRT started).

\section{Conflicts of interest}

None declared.

\section{References}

1 Chan L, Chaudhary K, Saha A et al. AKI in hospitalized patients with COVID-19. J Am Soc Nephrol 2020, in press (DOI: 10.1681/ ASN.2020050615).

2 Yang X, Jin Y, Li R et al. Prevalence and impact of acute renal impairment on COVID-19: a systematic review and meta-analysis. Crit Care 2020;24:356.

3 Wang L, Li X, Chen $\mathrm{H}$ et al. Coronavirus disease 19 infection does not result in acute kidney injury; an analysis of 116 hospitalised patients from Wuhan, China. Am J Nephrol 2020;51:343-8. 